\section{Electro-optic tunable band- pass filter based on long-period-grating-assisted asymmetric waveguide coupling}

\author{
Xuejun Lu, ${ }^{\text {a }}$ Miao Li, ${ }^{\text {b }}$ Rohit Samarth, ${ }^{c}$ and Lei Zheng ${ }^{a}$ \\ aniversity of Massachusetts Lowell, Department of \\ Electrical and Computer Engineering, Lowell, \\ Massachusetts 01854 \\ E-mail: xuejun_lu@uml.edu \\ ${ }^{b}$ People's Insurance Company of China, Zhengzhou, \\ China 450003 \\ 'University of Massachusetts Lowell, Department of \\ Physics, Lowell, Massachusetts 01854
}

\begin{abstract}
We present a new electro-optic (EO) tunable bandpass filter design based on a long-period-gratingassisted asymmetric waveguide coupling mechanism. A narrow passband width of $<0.2 \mathrm{~nm}$ and a large wavelength tuning range exceeding $30 \mathrm{~nm}$ can be obtained at a low driving voltage of $\sim 16 \mathrm{~V}$. This type of EO tunable filter would form key building blocks in dynamic wavelength division multiplexing (WDM) optical networks. () 2007 Society of Photo-Optical Instrumentation Engineers.
\end{abstract}

[DOI: 10.1117/1.2721420]

Subject terms: electro-optics; tunable optical filter; wavelength division multiplexing (WDM); asymmetric waveguide coupling; longperiod grating; waveguide mode coupling; integrated optics.

Paper 060634LR received Aug. 16, 2006; revised manuscript received Jan. 17, 2007; accepted for publication Jan. 26, 2007; published online Apr. 30, 2007.

Dense wavelength division multiplexing (WDM) is becoming a leading technology in fiber-optic networks. ${ }^{1-3}$ Dynamic WDM technologies with reconfigurable channels, bandwidth, and network topologies are expected to support aggregate bandwidth and low latency requirements of both civilian and military applications such as Internet access, high-quality videoconferencing, and information acquiring and sharing in aerospace. Tunable optical filters are among the key devices in realizing the dynamic WDM networks due to their capability of providing various dynamic functions such as wavelength tunable receivers, optical channel/ wavelength selections, and reconfigurations. ${ }^{3,4}$ To achieve these functionalities, general requirements for tunable optical filters include a large wavelength tuning range covering all WDM channels, low insertion loss, narrow passband, low polarization-dependent loss (PDL), and low channel cross talk. Existing electro-optic (EO) tunable optical filter technologies include fiber Fabry-Perot (F-P) interferometers, arrayed waveguide gratings (AWG), liquid crystal F-P interferometers, Mach-Zehnder interferometers, acousto-optic filters, and fiber Bragg gratings (FBG). ${ }^{4-9}$ While these optical filters have been employed in various optical networks, however, the major limitation is the small wavelength tuning range of a few nm, mainly due to the

0091-3286/2007/\$25.00 @ 2007 SPIE small EO coefficients $(<90 \mathrm{pm} / \mathrm{V})$ of existing materials, such as $\mathrm{LiNbO}_{3}$ and EO polymers. ${ }^{3,10,11}$ The small wavelength tuning range makes these devices unsuitable for broadband channel selections and reconfigurations across the whole WDM wavelength range (for example, C-band, $1530 \mathrm{~nm}$ to $1565 \mathrm{~nm}$ ). In this paper, we present a new EO filter structure based on a long-period-grating-assisted asymmetric waveguide coupling mechanism. An ultra-large wavelength tuning range exceeding $30 \mathrm{~nm}$ in the C-band $(1530 \mathrm{~nm}$ to $1565 \mathrm{~nm})$ and a narrow passband of $<0.2 \mathrm{~nm}$ can be achieved at a low driving voltage $\sim 16 \mathrm{~V}$ with a low channel cross talk of $\sim-25 \mathrm{~dB}$.

The cross section and top views of the EO tunable filter are shown in Figs. 1(a) and 1(b), respectively. The EO tunable filter consists of an input waveguide, an output waveguide, and a pair of coplanar EO tuning electrodes on top of the input waveguide. The input waveguide and the electrodes are separated by an $\mathrm{SiO}_{2}$ cladding layer. The waveguides are Ti-diffused $\mathrm{LiNbO}_{3}$ waveguides on an $\mathrm{X}$-cut $\mathrm{LiNbO}_{3}$ substrate. The input and output waveguides are asymmetric so that the coupling of optical signals between the two waveguides are phase-mismatched. The input waveguide has a long-period grating to generate an addition $k$ vector to provide phase-matched coupling for a certain wavelength. The phase-matching condition for optical coupling can be written as ${ }^{12,13}$ :

$\frac{2 \pi}{\lambda_{0}} n_{e f f, i n}-\frac{2 \pi}{\Lambda}=\frac{2 \pi}{\lambda_{0}} n_{e f f, o u t}$,

where $\lambda_{0}$ is the specific wavelength that meets the phasematch condition, $\Lambda$ is the period of the grating, and $n_{\text {eff,in }}$ and $n_{\text {eff,out }}$ are the effective indices of the input and output waveguide, respectively. The grating period $\Lambda$ is designed to be $\sim 10 \mu \mathrm{m}$, which is significantly larger than that of conventional quarter-wavelength Bragg gratings. From Eq. (1), the phase-matched wavelength $\lambda_{0}$ can be expressed as:

$\lambda_{0}=\left(n_{\text {eff,in }}-n_{\text {eff,out }}\right) \Lambda$.

The tuning of phase-matched wavelength $\lambda_{0}$ can thus be achieved by electro-optically changing the effective index of the input waveguide $n_{\text {eff,in }}$. As shown in Fig. 1(a), the bias voltage would generate an electric field along the $z$ direction. The EO-induced refractive index tuning can therefore be written as:

$\Delta n_{e f f}=-\frac{1}{2} n_{e f f}^{3} \gamma_{33} E_{z}$

where $\gamma_{33} \approx 31 \mathrm{pm} / \mathrm{V}$ is the $\mathrm{EO}$ coefficient of $\mathrm{LiNbO}_{3}$ along the $z$ direction (as shown in Fig. 1). The wavelength tuning can thus be written as:

$\Delta \lambda_{0}=\Delta n_{e f f, i n} \Lambda=\left(-\frac{1}{2} n_{e, e f f, i n}^{3} \gamma_{33} \frac{V}{d}\right) \Lambda$,

where $V$ is the applied tuning voltage, and $d$ is the effective separation of the top electrodes. The wavelength tuning ranges as a function of applied bias voltage for different grating periods are shown in Fig. 2 with parameters $d$ $=0.8 \mu \mathrm{m}$ and $n_{e, e f f}=2.13$ for $\mathrm{LiNbO}_{3}$ at the wavelength of $1.5 \mu \mathrm{m}$. As shown in Fig. 2, for the grating period $\Lambda$ 


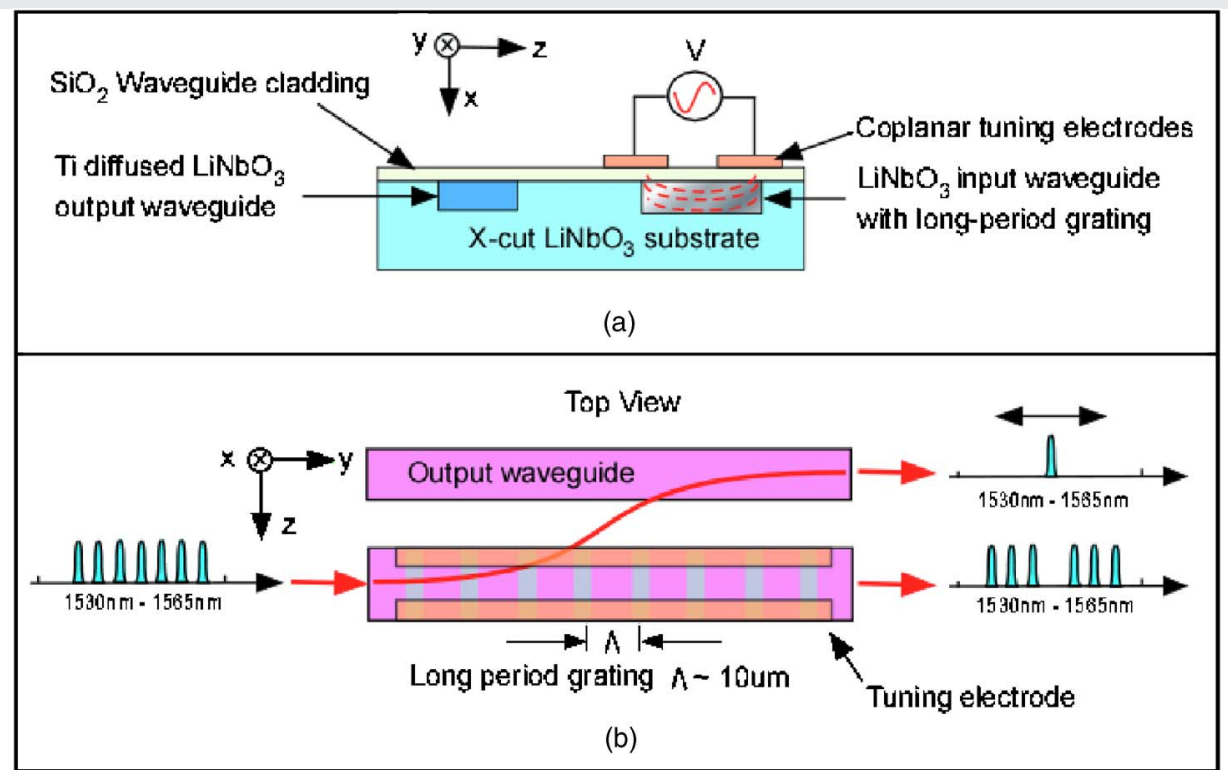

Fig. 1 Schematic structure of the EO tunable filter based on long-period-grating-assisted asymmetric coupling: (a) cross section; (b) top view.

$\sim 10 \mu \mathrm{m}$, an ultra-large wavelength tuning range of over $30 \mathrm{~nm}$ can be obtained at a low tuning voltage of $\sim 16 \mathrm{~V}$. Such a large tuning range enhancement factor overcomes the low EO coefficient limit of the conventional EO materials and allows the EO tunable filter to cover the whole C-band.

The phase mismatch $\Delta \beta$ for the wavelength away from the central wavelength can be written as ${ }^{12,13}$ :

$\Delta \beta=\frac{2 \pi}{\lambda^{\prime}} n_{e f f, i n}-\frac{2 \pi}{\lambda^{\prime}} n_{e f f, o u t}-\frac{2 \pi}{\Lambda}=2 \pi \cdot\left(\frac{\Delta \lambda}{\lambda^{\prime} \Lambda}\right)$,

where $\Delta \lambda=\lambda^{\prime}-\lambda_{0}$ is the wavelength detuning. The output power $P$ of the phase-mismatched coupling can be written as:

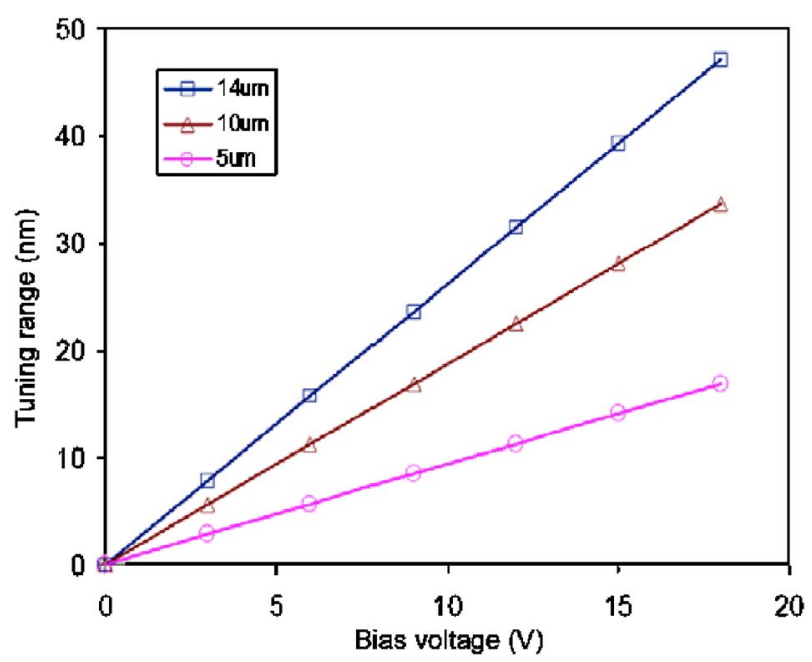

Fig. 2 Wavelength tuning range of the EO tunable filter as a function driving voltage for different grating periods.

$$
\begin{aligned}
P / P_{0} & =\frac{|\kappa|^{2}}{|\kappa|^{2}+\left(\frac{\Delta \beta}{2}\right)^{2}} \sin ^{2}\left[|\kappa|^{2}+\left(\frac{\Delta \beta}{2}\right)^{2}\right]^{1 / 2} L, \\
& =\frac{|\kappa|^{2}}{|\kappa|^{2}+\left(\frac{\pi \Delta \lambda}{\lambda^{\prime} \Lambda}\right)^{2}} \sin ^{2}\left[|\kappa|^{2}+\left(\frac{\pi \Delta \lambda}{\lambda^{\prime} \Lambda}\right)^{2}\right]^{1 / 2} L,
\end{aligned}
$$

where $L$ is the length of the asymmetric coupling, and $P$ and $P_{0}$ are the output powers for the phase-mismatched and phase-matched coupling, respectively. Figure 3 shows the passband profiles of two adjacent 0.8-nm-spaced WDM channels with parameters $\kappa=0.15 \mathrm{~cm}^{-1}, \Lambda=10 \mu \mathrm{m}$, and $L$ $=7.5 \mathrm{~cm}$. As illustrated in Fig. 3, the filter shows a narrow

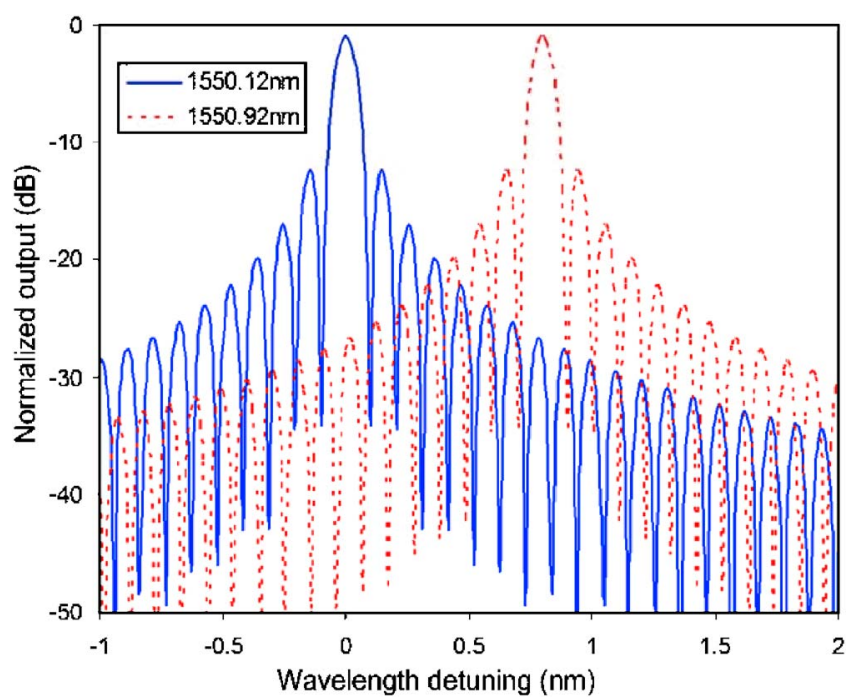

Fig. 3 Simulation results of the passband profile and cross talk of the EO tunable filter. 
passband of $\sim 0.2 \mathrm{~nm}$. The first sidelobes are located $\sim 0.15 \mathrm{~nm}$ from the main peak, with a sidelobe suppression ratio (SLSR) of $\sim 10.5 \mathrm{~dB}$. For the sidelobes that are $0.8 \mathrm{~nm}$ from the main peak, a high SLSR of $>25 \mathrm{~dB}$ can be obtained. Since ITU-Grid WDM channels have 0.8-nmwavelength spacing, a low cross talk of $<-25 \mathrm{~dB}$ can be expected. Higher SLSR can be obtained by reducing the grating period $\Lambda$, which would increase the tuning voltage accordingly. The total insertion of the device consists of the coupling loss $(\sim 0.5 \mathrm{~dB}$ from Fig. 3$)$ and the waveguide loss, which typically ranges from $0.4 \mathrm{~dB} / \mathrm{cm}$ to $1.0 \mathrm{~dB} / \mathrm{cm}$ at the wavelength of $1.5 \mu \mathrm{m} .{ }^{14}$ The total device insertion loss is therefore less than $8 \mathrm{~dB}$.

In conclusion, we present an EO tunable filter structure based on long-period-grating-assisted asymmetric waveguide coupling. This type of EO tunable filter overcomes the low EO coefficient limitation of convention materials and offers a large wavelength tuning range exceeding $30 \mathrm{~nm}$ at a low driving voltage $\sim 16 \mathrm{~V}$. It is expected to enable fast wavelength selection, communication channel reconfiguration, and packet- or cell-level switching for highly dynamic WDM optical networks.

\section{References}

1. J. P. Ryan, "WDM North American development trends," IEEE Commun. Mag. 36(2), 40-44 (1998).

2. T. Segawa, S. Matsuo, Y. Ohiso, T. Ishii, Y. Shibata, and H. Suzuki, "Fast tunable optical filter using cascaded Mach-Zehnder interferom- eters with apodized sampled gratings," IEEE Photonics Technol. Lett. 17, 139-141 (2005).

3. D. Sadot and E. Boimovich, "Tunable optical filters for WDM networks," IEEE Commun. Mag. 36(12), 50-55 (1998).

4. Y. Hibino, "Recent advances in high-density and large-scale AWG multi/demultiplexers with higher index-contrast silica-based PLCs," IEEE J. Sel. Top. Quantum Electron. 8, 1090-1101 (2002).

5. J. Stone and L. W. Stulz, "Pigtailed high-finesse tunable Fabry-Perot interferometer with large, medium, and small free spectral ranges," Electron. Lett. 23, 781-783 (1987).

6. J. L. Jackel, J. E. Baran, A. d'Alessandro, and D. A. Smith, "A passband-flattened acousto-optic filter," IEEE Photonics Technol. Lett. 7, 318-320 (1995).

7. J. Y. Liu and K. M. Johnson, "Analog smectic ferroelectric liquid crystal Fabry Perot optical tunable filter," IEEE Photonics Technol. Lett. 7, 1309-1311 (1995).

8. E. L. Wooten, R. L. Stone, E. W. Miles, E. M. Bradley, "Rapidly tunable narrowband wavelength filter using LiNb03 unbalanced Mach-Zehnder interferometers," J. Lightwave Technol. 14, 25302536 (1996).

9. A. Locco, H. G. Limberger, and R. P. Salathe, "Bragg grating fast tunable filter," Electron. Lett. 33, 2147-2148 (1997).

10. M.-C. Oh, H. Zhang, C. Zhang, H. Erlig, Y. Chang, B. Tsap, D Chang, A. Szep, W. H. Steier, H. R. Fetterman, and L. R. Dalton, "Recent Advances in electrooptic polymer modulators incorporating highly nonlinear chromophore," IEEE J. Sel. Top. Quantum Electron. 7, 826-835 (2001)

11. W. H. Steier and H.-C. Song et al., "Advances in polymer waveguide devices," in Proc. 16th Annual Meeting of the IEEE Lasers and Electro-Optics Society (LEOS 2003), Vol. 2, pp. 748-749 (2003).

12. A. Yariv, "Coupled-mode theory for guided-wave optics," IEEE J. Quantum Electron. 9, 919-933 (1973).

13. A. Yariv and P. C. Yeh, Optical Waves in Crystals, Wiley, New York (1984).

14. K. Noguchi, O. Mitomi, H. Miyazawa, and S. Seki, "A broadband Ti: $\mathrm{LiNbO}_{3}$ optical modulator with a ridge structure," J. Lightwave Technol. 13, 1164-1168 (1995). 